\title{
Ultra-sonografia da aorta abdominal e de seus ramos em cães
}

\author{
Ultrasonography of abdominal aorta and its branches in dogs
}

\author{
Lilian Kamikawa ${ }^{\mathrm{I}}$ Pedro Primo Bombonato ${ }^{\mathrm{II}}$
}

\section{RESUMO}

O ultra-som bidimensional e o ultra-som Doppler foram utilizados para avaliar a biometria e a hemodinâmica da aorta abdominal e artérias ilíacas externas de 131cães clinicamente normais. Os resultados da avaliação biométrica da aorta abdominal indicaram um diâmetro médio de $0,80 \mathrm{~cm}$ para o seu segmento diafragmático (AOD); $0,74 \mathrm{~cm}$ para o seu segmento caudal às artérias renais $(A O R)$ e $0,69 \mathrm{~cm}$ para o segmento cranial à sua bifurcação (AOT). A artéria ilíaca externa direita (AIED) apresentou o diâmetro médio de $0,42 \mathrm{~cm}$ e a artéria ilíaca externa esquerda (AIEE) o diâmetro médio de 0,39. O estudo hemodinâmico da aorta abdominal apresentou velocidade de pico sistólico médio de $104,00 \mathrm{~cm} \mathrm{~s}^{-1}$ para AOR; $99,61 \mathrm{~cm} \mathrm{~s}^{-1}$ para AOT; 85,47 $\mathrm{cm} \mathrm{s}^{-1}$ para AIED e 99,51 $\mathrm{cm} \mathrm{s}^{-1}$ para AIEE. Verificaram-se correlações de diferentes intensidades entre os diâmetros vasculares em diferentes pontos de tomadas e os fatores biométricos corpóreos (CRL). Correlações baixas foram observadas quando esses diâmetros foram confrontados com a idade.

Palavras-chave: ultra-sonografia, aorta, cães.

\section{ABSTRACT}

A duplex ultrasound system incorporating a pulsed wave Doppler ultrasound probe with conventional B-mode real-time imaging was used to evaluate the biometric and the hemodynamic of abdominal aorta and external iliac arteries of a hundred and thirty one normal dogs. Results of biometrics of abdominal aorta, presented a mean diameter of $0.80 \mathrm{~cm}$ in its diaphragmatic segment $(A O D) ; 0.74 \mathrm{~cm}$ in its segment caudal to the renal arteries $(A O R)$ and $0.69 \mathrm{~cm}$ in the segment cranial to its termination (AOT). The right-external-iliac artery (AIED) presented a mean diameter of $0.42 \mathrm{~cm}$ and the left-external iliac artery (AIEE) a mean diameter of $0.39 \mathrm{~cm}$. The hemodynamic study of the abdominal aorta presented a medium systolic peak velocity to AOR of $104 \mathrm{~cm}$ per sec.; to AOT of $99.61 \mathrm{~cm}$ per sec.; to AIED of $85.47 \mathrm{~cm}$ per sec. and to AIEE of $99.51 \mathrm{~cm}$ per sec. Correlations of different intensities between the vascular diameters have been verified between the measured points and the corporeal biometric factors (Crown RumpLenght). Low correlations have been observed regarding the diameters in relation to the age.

Key words: ultrasonography, aorta, dogs.

\section{INTRODUÇÃO}

A técnica de ultra-som bidimensional fornece informações dinâmicas relativas à anatomia e à morfologia vascular, sendo complementada pela técnica de ultra-som Doppler de onda pulsátil, que permite detectar e quantificar a presença, a direção, a velocidade e as características do fluxo sangüíneo nos vasos (TAYLOR, 1990; CERRI \& ROCHA, 1993; SZATMÁRI et al., 2001).

De uma forma não-invasiva, a combinação das técnicas de ultra-som bidimensional e ultra-som Doppler de onda pulsátil em um único aparelho (duplex Doppler) permite uma avaliação precisa e rápida do estado físico das paredes vasculares (imagens dinâmicas) associada às características do fluxo sangǘneo dos principais vasos, podendo ser utilizada em qualquer idade, não necessitando de contraste, radiações ionisantes e anestesia (FERNER, 1994)

O conhecimento da localização e das características morfológicas e hemodinâmicas tem fundamental importância na identificação dos vasos sangüíneos, pois as suas características ao ultra-som Doppler são praticamente específicas (SZATMÁRI et al., 2001). Dessa forma, a possibilidade de usufruir de parâmetros normais em animais sadios permite observar

\footnotetext{
'Departamento de cirurgia, Universidade de São Paulo (USP). Rua Adolfo Lutz, n.123, Vila Apiaí, 09185-400, Santo André, São Paulo, Brasil. E-mail: likamik@uol.com.br. Autor para correspondência.

"Departamento de cirurgia, área de concentração anatomia dos animais domésticos e silvestres, USP, Santo André, SP.
} 
possíveis alterações vasculares, possibilitando obterse critérios confiáveis para um diagnóstico e prognóstico (VALENTINI et al., 1996).

Nesse contexto, considera-se que o ultrasom bidimensional e o ultra-som Doppler podem ser empregados como técnicas de auxílio diagnóstico por imagem de eleição para o estudo morfométrico, morfológico e hemodinâmico da aorta abdominal e de seus principais ramos.

Considerando esses fatos, propôs-se o presente estudo, utilizando cães de diferentes raças, idades e sexo, clinicamente normais, tendo como objetivos obter a biometria da aorta abdominal e das artérias ilíacas externas direita e esquerda, avaliar a hemodinâmica destes vasos e realizar as correlações entre biometria dos vasos, fatores biométricos corpóreos (Crown-Rump Lenght) e idade.

\section{MATERIAL E MÉTODOS}

Constam do estudo morfométrico da aorta abdominal e seus ramos 131 cães, sendo 35 machos e 96 fêmeas, de raças variadas e idade entre 3 e 192 meses (média de idade de 76 meses). Os cães foram divididos em seis grupos, de acordo com os fatores biométricos corpóreos “Crown-Rump Lenght” (CRL), sendo que o comprimento do animal foi tomado da região occipital até a região de transição sacro-coccígea.

O Grupo I foi composto por 13 animais, sendo 3 machos e 10 fêmeas, com CRL entre 30 e $40 \mathrm{~cm}$ de comprimento. O Grupo II foi composto por 23 animais, sendo 4 machos e 19 fêmeas, com CRL entre 41 e 50cm de comprimento. O Grupo III foi composto por 50 animais, sendo 11 machos e 39 fêmeas, com CRL entre 51 e $60 \mathrm{~cm}$ de comprimento. O Grupo IV foi composto por 29 animais, sendo 12 machos e 17 fêmeas, com CRL entre 61 e $70 \mathrm{~cm}$ de comprimento. O Grupo V foi composto por 11 animais, sendo 4 machos e 7 fêmeas, com CRL entre 71 e $80 \mathrm{~cm}$ de comprimento. O Grupo VI foi composto por 5 animais, sendo 1 macho e 4 fêmeas, com CRL superior a $81 \mathrm{~cm}$ de comprimento.

Para o estudo hemodinâmico da aorta abdominal, foram selecionados 39 cães, 13 machos e 26 fêmeas, de raças variadas e idade entre 3 e 168 meses (idade média de 81 meses). Dentre esses 39 animais, foram selecionados 27 cães (8 machos e 19 fêmeas) para o estudo hemodinâmico das artérias ilíacas externas direita e esquerda.

Todos os cães submetidos ao estudo hemodinâmico da aorta abdominal e das artérias ilíacas externas apresentavam temperamento dócil, não apresentavam histórico prévio de alterações cardiovasculares e alterações ao exame ultrasonográfico abdominal.
Descrição da técnica do exame ultra-sonográfico

Para a coleta de informações sobre a morfometria da aorta abdominal e de seus principais ramos, utilizou-se o aparelho de ultra-som bidimensional $^{\text {a }}$, com um transdutor microconvexo de 5.0Mhz e um transdutor linear de 7.5Mhz. Todos os vasos foram visibilizados ao corte longitudinal e a mensuração foi realizada neste plano, posicionandose os marcadores eletrônicos “calipers” nas superfícies internas da parede dorsal-ventral ou latero-lateral do vaso durante a fase diastólica, quando o vaso apresentava o seu maior diâmetro. O diâmetro do vaso foi tomado em centímetros.

A aorta abdominal foi mensurada nas regiões diafragmática (AOD) caudalmente às artérias renais direita e esquerda (AOR) e cranialmente à bifurcação (AOT). (Figura 1). As artérias ilíacas externas direita e esquerda foram mensuradas imediatamente distal à bifurcação da aorta abdominal.

Para a coleta de informações hemodinâmicas e biométricas da aorta abdominal e artérias ilíacas externas, foi utilizado o aparelho de ultra-som Doppler ${ }^{\mathrm{b}}$, com um transdutor convexo de 3.5Mhz e um transdutor linear de 7.5Mhz. Neste aparelho, o estudo com ultrasom Doppler de onda pulsátil foi realizado com programação técnica do equipamento para abdome. Os ajustes dos parâmetros técnicos para a análise foram baseados no protocolo recomendado por OLIVEIRA (1997), utilizando-se a máxima potência em Doppler pulsado, o menor filtro de parede para a caracterização de toda a amplitude do sinal, a amostra de volume (região onde os sinais Doppler são medidos), localizada no centro do vaso para a reprodução do fluxo laminar de maior amplitude, e o ângulo entre o transdutor e a amostra de no máximo $60^{\circ}$.

A documentação e impressão fotográfica dos exames foi realizada em Video Grafic Printer ${ }^{c}$.

\section{RESULTADOS}

\section{Aspectos biométricos}

A aorta abdominal apresenta uma diminuição de seu diâmetro na direção crânio-caudal. O diâmetro da aorta abdominal da amostra geral em seu segmento diafragmático (AOD) variou de 0,60 a 1,10cm, com média de $0,80 \mathrm{~cm}$ e desvio padrão de 0,15 . Para o segmento caudal às artérias (AOR), o seu diâmetro variou de 0,48 a 1,30cm, com média de 0,74cm e desvio padrão de 0,20. Para o segmento cranial à sua bifurcação (AOT), o seu diâmetro variou de 0,30 a $1,40 \mathrm{~cm}$, com média de $0,69 \mathrm{~cm}$ e desvio padrão de 0,18 .

Não houve uma diferença significativa entre a média do diâmetro das artérias ilíacas externas. O 


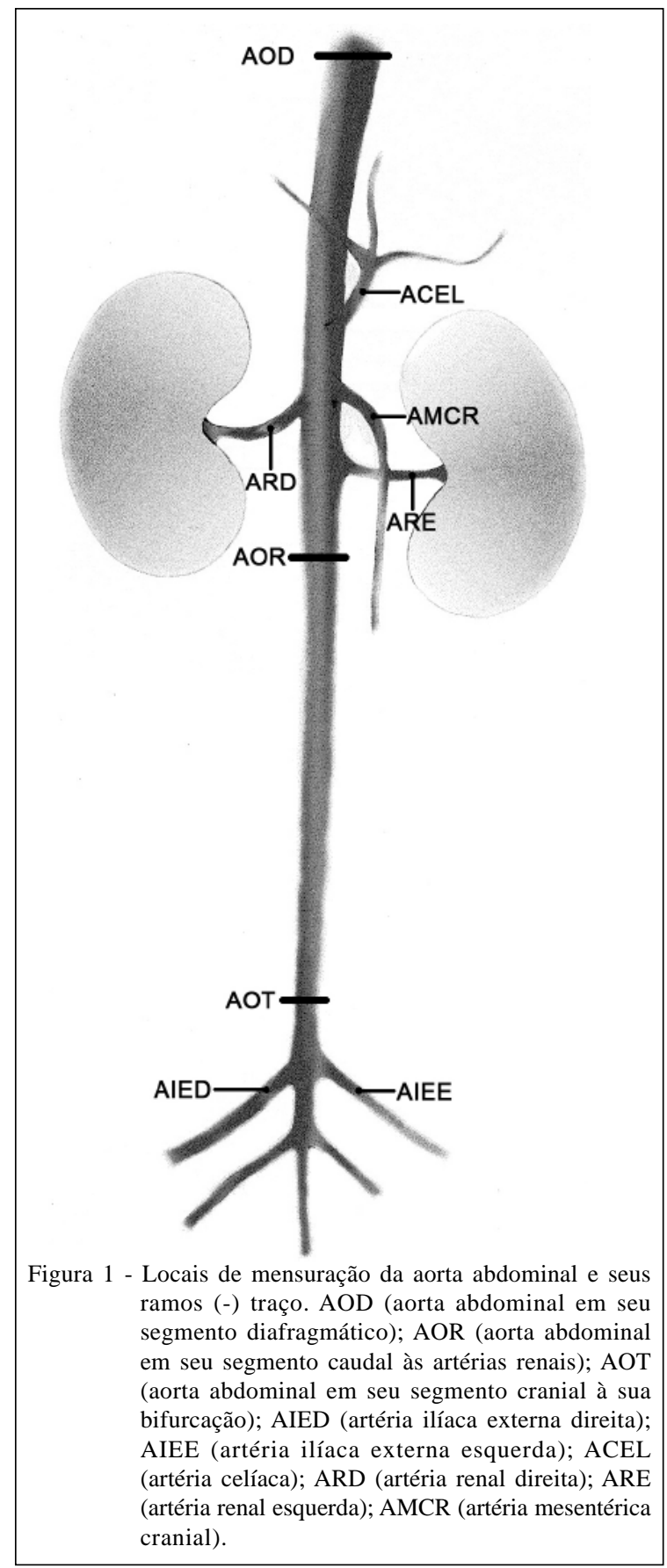

diâmetro da artéria ilíaca externa direita (AIED) variou de 0,27 a $0,70 \mathrm{~cm}$, com média de $0,42 \mathrm{~cm}$ e desvio padrão de 0,13 . O diâmetro da artéria ilíaca externa esquerda (AIEE) variou de 0,20 a $0,80 \mathrm{~cm}$, com média de $0,39 \mathrm{~cm}$ e desvio padrão de 0,11 .

Quando submetida a amostra geral a uma análise de correlação entre o valor obtido do diâmetro médio de um segmento da aorta abdominal e o diâmetro médio dos vasos localizados caudalmente a ela, foi observada uma correlação de moderada a alta intensidade, com valores variando de 0,49 a 0,96 .

O diâmetro da aorta abdominal em seu segmento diafragmático (AOD), quando confrontado com o diâmetro da aorta abdominal em seus segmentos caudal às artérias renais direita e esquerda (AOR) e cranial à bifurcação (AOT), apresentaram ambos a mesma correlação de 0,77 . Quando este mesmo segmento foi confrontado com as artérias ilíacas externas direita (AIED) e esquerda (AIEE), os valores de correlação encontrados foram, respectivamente, de 0,66 e 0,49 .

Já o diâmetro do segmento da aorta abdominal caudal às artérias renais direita e esquerda (AOR) foi confrontado com o diâmetro do segmento da aorta abdominal cranial à bifurcação (AOT), artéria ilíaca externa direita (AIED) e artéria ilíaca externa esquerda (AIEE), sendo verificada uma correlação de alta intensidade. Os valores obtidos foram, respectivamente, de 0,95, 0,96 e 0,93.

O CRL da amostra geral, independentemente dos fatores biométricos corpóreos, foi confrontado com os segmentos da aorta abdominal e seus ramos, sendo obtidos valores desta correlação de moderada a alta intensidade $(r>0,70)$.

Foi observada correlação de baixa e moderada intensidade quando foi confrontado o diâmetro da aorta abdominal em seu segmento cranial a sua bifurcação (AOT) dos diferentes grupos CRL com a idade $(r<0,40)$.

\section{Aspectos hemodinâmicos}

Nos cães examinados, a onda da aorta abdominal nos segmentos caudal às artérias renais direita e esquerda (AOR) e cranial à bifurcação (AOT) e artérias ilíacas externas apresentou forma trifásica (Figura 2A, B).

Considerando os animais examinados independentemente dos fatores biométricos corpóreos (CRL), da idade e do sexo, a amostra geral indicou que a velocidade de pico sistólico para o segmento caudal às artérias renais (AOR) variou de 77,50 a 150,00 $\mathrm{cm} \mathrm{s}^{-1}$, com média de $104,18 \mathrm{~cm} / \mathrm{s}$ e desvio padrão de 22,04 . Para o segmento da aorta abdominal cranial à bifurcação foram observados valores de velocidade de pico sistólico entre 76,00 a $130,00 \mathrm{~cm} \mathrm{~s}^{-1}$, com média de $99,61 \mathrm{~cm} \mathrm{~s}^{-1}$ e desvio padrão de 15,46.

Neste estudo, as ondas espectrais das artérias ilíacas externas direita e esquerda observadas nos cães examinados apresentaram forma trifásica (Figura 2 C, D). Considerando os animais examinados, independentemente dos fatores biométricos corpóreos (CRL), da idade e do sexo, a amostra geral indicou que 


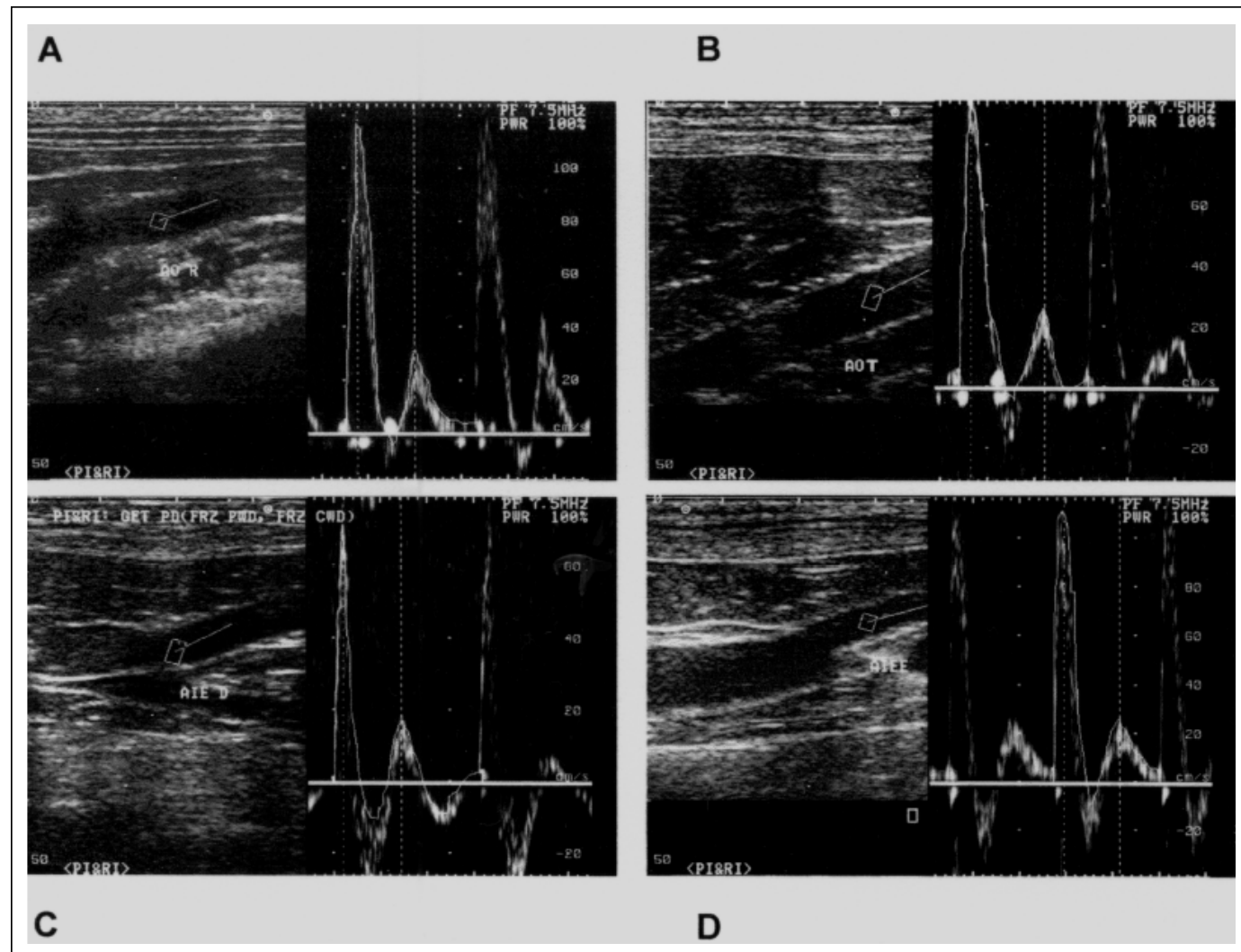

Figura 2 - A. espectro de onda de fluxo sangüíneo em aorta abdominal em seu segmento caudal às artérias renais (AOR); onda do tipo trifásica.

B. espectro de onda de fluxo sangüíneo em aorta abdominal em seu segmnto cranial à sua bifurcação (AOT); onda do tipo trifásica.

C: espectro de onda de fluxo sangüíneo em artéria ilíaca externa direita (AIED); onda do tipo trifásica. D: espectro de onda de fluxo sangüíneo em artéria ilíaca externa esquerda (AIEE); onda do tipo trifásica.

a velocidade de pico sistólico para a artéria ilíaca externa direita variou de 61,90 a $110,00 \mathrm{~cm} \mathrm{~s}^{-1}$, com média de $85,47 \mathrm{~cm} \mathrm{~s}^{-1}$ e desvio padrão de 13,69. Para a artéria ilíaca externa esquerda, foram observados valores de velocidade de pico sistólico entre 66,90 e $110,00 \mathrm{~cm} \mathrm{~s}^{-1}$, com média de $99,51 \mathrm{~cm} \mathrm{~s}^{-1}$ e desvio padrão de 14,33.

\section{DISCUSSÃO}

Na literatura compulsada, foi evidenciado reduzido número de trabalhos que tratam sistematicamente da biometria e da hemodinâmica da aorta abdominal e de seus ramos ao exame ultrasonográfico. A introdução de técnicas mais precisas, como o ultra-som modo B e ultra-som Doppler, tem possibilitado efetivos avanços para os diagnósticos de moléstias vasculares; no entanto, a literatura é escassa e parcimoniosa na indicação de "valores normais" que possam servir de valores de referência. Segundo FINN-BODNER \& HUDSON (1998), o diâmetro normal da aorta para animais de diferentes tamanhos ainda não foi bem estabelecido, motivo pelo qual empreendeu-se tal pesquisa, e os autores sugerem que o diâmetro médio para cães de 18 a $23 \mathrm{Kg}$ seja de $1,0 \mathrm{~cm}$, fato este próximo daqueles observados no presente trabalho.

Baseando-se nas escassas informações biométricas relatadas até agora, procurou-se, neste estudo, mensurar o diâmetro normal da aorta abdominal em três segmentos distintos, sendo a avaliação dos resultados realizada de duas formas; pela amostra geral, independentemente do fator biométrico corpóreo (CRL) e pela divisão dos cães em grupos de acordo com o fator biométrico corpóreo (CRL). Pelos resultados obtidos, constatou-se que a aorta abdominal estreitase gradualmente no sentido crânio-caudal, como relataram BLUTH(1984); FINN-BODNER \& HUDSON (1998); CERRI \& ROCHA (1993); COELHO (1993). Somente no grupo VI, tal constatação não foi confirmada, sendo o diâmetro médio do segmento caudal às artérias renais (AOR) superior ao segmento

Ciência Rural, v.37, n.2, mar-abr, 2007. 
diafragmático (AOD), o que pode ter sido determinado pelo tamanho de amostragem nesse grupo.

A correlação entre o fator biométrico corpóreo (CRL) da amostra geral e o diâmetro da aorta abdominal e de seus ramos indicou intensidade de moderada a alta . Os dados coletados indicaram que a biometria da aorta abdominal e de seus ramos relacionase de uma forma positiva e significativa com o CRL, sinalizando claramente que, quanto maior o animal, maior os diâmetros vasculares, mesmo quando considerados estreitos intervalos de classe associados às medidas do CRL, fato executado quando da subdivisão em 6 grupos.

Foi observada correlação de baixa e média intensidade quando confrontaram-se os diferentes grupos, que indicavam animais de tamanhos diferentes, com a idade, de tal forma que os dados coletados indicaram que a biometria da aorta abdominal na bifurcação está relacionada ao tamanho dos animais e não à sua idade. Isso reforça a informação lógica de que, por exemplo, um animal pequeno e idoso não necessariamente apresenta, por conta da idade, maior diâmetro da aorta abdominal. Fato este não observado na medicina humana, pois, segundo COELHO (1993), a aorta abdominal sofre um alargamento gradual com a idade. A partir dessas informações, surge a presente questão: estariam os cães menos predispostos a lesões vasculares de aorta abdominal em decorrência desta baixa correlação entre a idade e o diâmetro do vaso?

A correlação entre a idade e o diâmetro do segmento da aorta cranial à bifurcação (AOT) demonstrou que, à medida que o tamanho da amostra aumentava, o resultado obtido na correlação tendia à diminuição. Portanto, as correlações entre a idade e os segmentos diafragmático (AOD) e caudal às artérias renais (AOR) não foram realizadas por conta do pequeno número de dados obtidos, o que poderia comprometer a acuracidade dos resultados.

À medida que a aorta abdominal diminuía 0 seu diâmetro crânio-caudalmente, ou seja, à medida que os valores do diâmetro da aorta mais próximos aos vasos correlacionados eram adquiridos, os valores absolutos de "r" aumentavam. Portanto, o “ $\mathrm{r}$ ” apresentou-se maior quanto mais calibroso e próximo era o ponto de tomada da emergência do vaso correlacionado, fato este que pode estar relacionado à proporção quadrática a que o calibre vascular está subordinado.

Pelos resultados obtidos da velocidade média de pico sistólico da amostra geral, nos segmentos da aorta abdominal caudal às artérias renais (AOR) com diâmetro médio de $0,72 \mathrm{~cm}$ e velocidade de $104,18 \mathrm{~cm}$ $\mathrm{s}^{-1}$, e na bifurcação com diâmetro médio de $0,68 \mathrm{~cm}$ e velocidade de $99,61 \mathrm{~cm} \mathrm{~s}^{-1}$, pode-se observar que a velocidade de fluxo sangüíneo é diretamente proporcional ao diâmetro do vaso, isto é, à medida que o calibre do vaso diminui, a velocidade de fluxo sangüíneo também diminui.

Tal fato também foi observado na artéria ilíaca externa direta, cujo diâmetro médio do vaso foi de $0,40 \mathrm{~cm}$ e a velocidade de pico sistólico mediu $83,90 \mathrm{~cm} \mathrm{~s}^{-1}$. Contudo, tal observação não demonstrou ser verdadeira para a artéria ilíaca externa esquerda, cujo diâmetro médio do vaso foi de $0,38 \mathrm{~cm}$ e a velocidade de pico sistólico mediu $99,51 \mathrm{~cm} \mathrm{~s}^{-1}$. Este fato poderia levar a suposição de que a proporcionalidade entre o diâmetro médio e a velocidade devesse também ser influenciado pela importância, dimensão, características e exigências nutricionais que o território vascular teria, especialmente quando comparou-se estes fatos no relativo às artérias ilíacas externas direita a esquerda, indicando que estes casos sofreriam e responderiam diretamente àquelas influências.

\section{CONCLUSÕES}

A partir dos dados obtidos, é possível concluir que o diâmetro médio da aorta abdominal e de seus ramos, na amostra geral, mensurados pelo ultrasom bidimensional, resultaram em $0,80 \mathrm{~cm}$ para AOD, 0,74cm para AOR, $0,69 \mathrm{~cm}$ para AOT, $0,42 \mathrm{~cm}$ para AIED e $0,39 \mathrm{~cm}$ para AIEE. Existem correlações positivas de média a alta intensidade, entre o CRL da amostra geral e o diâmetro da aorta abdominal e seus ramos; já correlações de média a baixa intensidade são identificadas entre o diâmetro de AOT e a idade dos diferentes grupos CRL.

Os valores médios hemodinâmicos da aorta abdominal e das artérias ilíacas externas indicaram velocidade de pico sistólico de $104,18 \mathrm{~cm} \mathrm{~s}^{-1}$ para AOR, $99,61 \mathrm{~cm} \mathrm{~s}^{-1}$ para AOT, 85,47 $\mathrm{cm} \mathrm{s}^{-1}$ para AIED e $99,51 \mathrm{~cm}$ $\mathrm{s}^{-1}$ para AIEE. A correlação é maior quanto mais calibroso e próximo forem os diâmetros da aorta abdominal e de seus ramos.

\section{COMITÊ DE ÉTICA E BIOÉTICA}

Projeto aprovado sob o número 224/2002 pela Comissão de Bioética da FMVZ-USP.

\section{FONTES DE AQUISIÇÃO}

aUltra-som bidimensional da marca Fukuda Denshi ${ }^{\circledR}$, modelo UF-4500.

bUltra-som Doppler da marca Fukuda Denshi ${ }^{\circledR}$, modelo UF7500 .

${ }^{c}$ Video graphic printer da marca Sony ${ }^{\circledR}$, modelo UP 850 MD. 


\section{REFERÊNCIAS}

BLUTH, E.I. Ultrasound of the abdominal aorta. Archives of Internal Medicine, v.144, p.377-380, 1984.

CERRI, G.C.; ROCHA, D.C. Ultra-sonografia abdominal. São Paulo: Sarvier, 1993. 459p.

COELHO, N.A. Ecografia e doppler vascular: aorta abdominal e seus ramos. Revista de Angiologia e Cirurgia Vascular, v.2, n.3, p.148-157, 1993

FERNER, M. Instrumentos doppler. Informativo da Sociedade Brasileira de Ultra-sonografia, v.1, n.5, p.610,1994

FINN-BODNER, S.T.; HUDSON, J.A. Abdominal vascular sonography. Veterinary Clinics of North America: Small Animal Practice, v.28, n.4, p.887-941, 1998.
OLIVEIRA, I.R.S. Ultra-sonografia com doppler aplicada ao rastreamento da estenose da artéria renal: estudo comparativo com angiografia. 1997. 137f. Tese (Doutorado em Radiologia) - Faculdade de Medicina da Universidade de São Paulo.

SZATMÁRI, V. et al. Normal duplex doppler waveforms of major abdominal blood vessels in dogs: a review. Veterinary Radiology \& Ultrasound, v.42, n.2, p.93-107, 2001.

TAYLOR, J.W.K. Doppler US. Part I. Basic principles, intrumentation, and pitfals. Radiology, v.174, n.2, p.297307, 1990.

VALENTINI, S. et al. Analisi ecografica di alcuni parametri fisiologici dell'aorta addominale del cane. Veterinaria, v.10, n.3, p.53-57, 1996 